\title{
Replicator Equations and Models of Biological Populations and Communities
}

\author{
G. P. $\operatorname{Karev}^{1}$ *, I. G. Kareva ${ }^{2}$ \\ ${ }^{1}$ National Center for Biotechnology Information, National Institute of Health, Bldg. 38A, Rm. \\ 5N511N, 8600 Rockville Pike, Bethesda, MD 20894, USA \\ ${ }^{2}$ Newman-Lakka Institute for Personalized Cancer Care, Floating Hospital for Children, Tufts Medical \\ Center, 75 Kneeland St., Boston, MA, 02111
}

\begin{abstract}
An overview of a general approach for mathematical modeling of evolving heterogeneous populations using a wide class of selection systems and replicator equations (RE) is presented. The method allows visualizing evolutionary trajectories of evolving heterogeneous populations over time, while still enabling use of analytical tools of bifurcation theory. The developed theory involves introducing escort systems of auxiliary "keystone" variables, which reduce complex multi-dimensional inhomogeneous models to low dimensional systems of ODEs that in many cases can be investigated analytically. In addition to a comprehensive theoretical framework, a set of examples of the method's applicability to questions ranging from preventing the tragedy of the commons to cancer therapy is presented.
\end{abstract}

Keywords and phrases: population heterogeneity, replicator equation, selection system, tragedy of the commons, cancer

Mathematics Subject Classification: 37N25, 92A30

\section{Introduction}

Evolving biological systems are by nature heterogeneous. In cases when variations in a particular trait directly affect fitness, such as in many ecological, financial or social systems, heterogeneity cannot be overlooked. A number of great advances have already been made in the field of mathematical modeling and analysis of dynamical systems $[2,4,38]$; however, parametrically homogeneous predictive models are unfortunately often of limited use, since in them one is usually not accounting for selective pressures that individuals within the population impose on each other in addition to the pressures that they experience from interactions with the environment.

There do already exist some ways of incorporating heritable individual-specific heterogeneity into dynamical systems. These approaches, among others, include introduction of additional self-similar equations, which increases system dimensionality [37]; separation of time scales, which breaks the system down into subsystems that change at different rates with respect to each other [32]; or introduction of stochasticity into the system [5], which could allow for the trajectories to escape the domain of attraction of equilibrium points or cycles in a deterministic model. While allowing incorporation of some hetero-

\footnotetext{
*Corresponding author. E-mail: karev@ncbi.nlm.nih.gov
} 
geneity in the model, these methods increase dimensionality of the system to an extent that disallows the possibility for use of any analytical results.

One of the examples, where visualization of transitional regimes that an evolving system goes through would be crucial, is when one is looking at differential depletion of shared resources, which, when not taken under control in a timely manner, can lead to what has become known as the tragedy of the commons $[6,16,47]$. The ability to identify transitional regimes [31], which would have served as warning signals, could provide an opportunity to prevent, or at least delay system collapse.

The paper is organized as follows: first we present a description and derivation of the proposed method, which we refer to as HKV, or "hidden keystone variables" method and which allows incorporating a very high degree of heritable heterogeneity into dynamical systems (noticeably, the method does not allow incorporating age or spatial heterogeneity, as these are not heritable characteristics). Then we reformulate a number of previously studied problems in such a way that now their analysis can be extended and deepened compared to what could be done previously. Finally, we present several specific examples from the fields of ecology and also cancer modeling, where the proposed method enabled formulation and investigation of questions that could not previously be addressed.

\section{Simplified version of the HKV method and models of Malthusian type}

Let us start from a simplified version of the model of inhomogeneous population and give two examples, which may clarify the main ideas of the developed approach. The examples give a motivation for focusing specifically on a wide class of models of evolving heterogeneous populations and corresponding replicator equations (RE). Furthermore, they provide a vivid illustration for how one can reduce the dimensionality of initially infinitely-dimensional system of ODEs through introducing auxiliary "keystone" variables (the terminology was chosen to parallel the notion of keystone species in ecological systems, whose impact on overall system dynamics is disproportionally large relative to their abundance).

Consider an inhomogeneous population composed of individuals with different reproduction rates (Malthusian parameters) $a$; we refer to the set of all individuals with the given value of parameter $a$ as an $a$-clone. Let $l(t, a)$ be the size of $a$-clone at the moment $t$.

In this example, we assume that the growth rate of each clone depends on the total population size $N(t)$. Dynamics of such a population can be described by the following model:

$$
\begin{aligned}
& \frac{d l(t, a)}{d t}=a l(t, a) g(N), \\
& N(t)=\int_{A} l(t, a) d a
\end{aligned}
$$

where $g(N)$ is some function, chosen depending on the specifics of each model. For example, if $g(N)=$ const, then (2.1) is an inhomogeneous Malthusian model; if $g(N)=\left(1-\frac{N}{C}\right)$, then (2.1) describes inhomogeneous model, where each clone grows logistically with a common carrying capacity $C$.

Denote $P(t, a)=l(t, a) / N(t)$; the probability density function (pdf) $P(t, a)$ describes the distribution of the parameter $a$ throughout the population at $t$ moment. Hereinafter we use the notation

$$
E_{t}[f]=\int_{0}^{\infty} f(a) P(t, a) d a
$$

for any integrable function $f(a)$ to refer to the expected value of $f$ at time $t$.

It can be easily proven and is known in a more general context [15] that the population size $N(t)$ satisfies the equation

$$
\frac{d N}{d t}=N E_{t}[a] g(N)
$$

and the pdf $P(t, a)$ solves the replicator equation of the form

$$
\frac{d P(t, a)}{d t}=P(t, a) g(N)\left(a-E_{t}[a]\right)
$$


Next, assume that the initial pdf $P(0, a)$ of the Malthusian parameter $a$ is given and its moment generating function (mgf)

$$
M_{0}(\lambda)=\int_{A} e^{\lambda a} P(0, a) d a
$$

is known. This information could be obtained either from data or in the absence of data, from general theoretical considerations.

In order to solve problem (2.1), let us define formally the "keystone" auxiliary variable $q(t)$ as the solution to the Cauchy problem

$$
\frac{d q}{d t}=g(N), q(0)=0
$$

This equation cannot be solved at this moment, because the population size $N(t)$ is unknown. However, the clone densities and population size can be expressed with the help of the keystone variable $q(t)$ :

$$
\begin{aligned}
& l(t, a)=l(0, a) e^{a q(t)}=N(0) P(0, a) e^{a q(t)}, \\
& N(t)=N(0) \int_{A} e^{a q(t)} P(0, a) d a=N(0) M_{0}(q(t)) .
\end{aligned}
$$

Now the equation for the auxiliary variable $q(t)$ can be written in a closed form

$$
\begin{aligned}
& \frac{d q}{d t}=g\left(N(0) M_{0}(q(t)),\right. \\
& q(0)=0 .
\end{aligned}
$$

Now we can completely solve the initial problem (2.1) and corresponding RE (2.3). The clone densities and population size are given by formulas (2.5).

The current parameter distribution $P(t, a)$ is determined by the formula

$$
P(t, a)=\frac{l(t, a)}{N(t)}=\frac{P(0, a) e^{a q(t)}}{M_{0}(q(t))} .
$$

The mgf of the current distribution $P(t, a)$

$$
M_{t}(\lambda)=E_{t}\left[e^{\lambda a}\right]=M_{0}(\lambda+q(t)) / M_{0}(q(t))
$$

and

$$
E_{t}[a]=\frac{d \ln M_{t}(q(t))}{d q(t)}
$$

We refer to the model (2.1) as a model of Malthusian type, and the variable $q(t)$ can be considered as the "internal time" of the model in the sense that with respect to the new time $q(t)$, each clone grows as if independently of other clones. Indeed, making the change of variables $d q \rightarrow g(N) d t$, we obtain from (2.5):

$$
\frac{d l(q(t), a)}{d t}=\frac{d l}{d q} \frac{d q}{d t}=\operatorname{alg}(N) .
$$

Therefore, $\frac{d l(q, a)}{d q}=a l(q, a)$, which is the standard Malthusian model, which describes a system of freegrowing clones.

\subsection{Example 1. Inhomogeneous Malthusian model}

Let us consider a case, where the reproduction rate of each clone is a constant. Then we obtain the simplest inhomogeneous Malthusian model of the form

$$
\frac{d l(t, a)}{d t}=a l(t, a) .
$$


The model describes a population of clones $l(t, a)$, each of which grows at its own rate, which does not depend on any other factors. The solution to this equation is

$$
l(t, a)=l(0, a) e^{a t} .
$$

Now let $M_{0}(\lambda)=\int_{A} e^{\lambda a} P(0, a) d a$ be the mgf of the initial distribution of the Malthusian parameter $a$. Then the total size of the population is

$$
N(t)=\int_{A} e^{\lambda a} P(0, a) d a=N(0) M_{0}(t) .
$$

The corresponding replicator equation reads

$$
\frac{d P(t, a)}{d t}=P(t, a)\left(a-E_{t}[a]\right)
$$

The escort equation is trivial in this case, $\frac{d q}{d t}=1$, and the keystone variable is just a time, so the solution to the replicator equation is

$$
P(t, a)=P(0, a) \frac{e^{a t}}{M_{0}(t)} .
$$

Moreover, the rate of change of the total population size is now

$$
\frac{d N}{d t}=N E_{t}[a]
$$

and the rate of change of the mean of $a$ satisfies

$$
\frac{d E_{t}[a]}{d t}=\operatorname{Var}_{t}[a]>0
$$

which incidentally is the simplest version of the Fisher Fundamental theorem of selection [8]. From this one can see that any inhomogeneous Malthusian population increases hyper-exponentially compared to exponential growth of a parametrically homogeneous Malthusian population (see $[22,27]$ for details and different examples).

Remark, that even the simplest inhomogeneous Malthusian model (2.9) and replicator equation (2.10) possess a variety of solutions depending on the initial distribution of clones in the population, and thus the predicted dynamics could be qualitatively different depending on the initial state of the heterogeneous population. Some examples will be considered in Section 4.

\subsection{Example 2. Logistic equation with distributed Malthusian parameter and the struggle for existence}

All populations have the capacity to grow exponentially under ideal conditions, and no population can grow exponentially forever - there are limits to growth, which is the underlying principle of the Malthusian Struggle for Existence [12]. Furthermore, according to Darwin, the struggle for existence implies the survival of the fittest. However, the following model shows that the situation may be more complex.

A simple conceptual model for Malthusian Struggle for Existence is given by inhomogeneous logistic equation, which accounts both for free exponential growth and for resource limitations [50]:

$$
\frac{d l(t ; a)}{d t}=a l(t ; a)\left(1-\frac{N}{C}\right)^{k}
$$

where $a$ is the Malthusian reproduction rate, which is assumed to be distributed, $C$ is the common carrying capacity, $N$ is the total population size, and $k>0$ is a fitting constant. Model (2.11) is a model of Malthusian type $(2.1)$ with $g(N)=\left(1-\frac{N}{C}\right)^{k}$. 
According to the theory described in Section 1.1, let us define the auxiliary keystone variable by the equation

$$
\frac{d q}{d t}=\left(1-\frac{N}{C}\right)^{k}, q(0)=0
$$

Then

$$
l(t, a)=l(0, a) e^{a q(t)}
$$

the total size of the population is given by the formula

$$
N(t)=N(0) M_{0}(q(t))
$$

where $M_{0}(\lambda)$ is the mgf of the initial distribution of the parameter $a$.

The population size $N(t)$ solves the non-autonomous logistic equation

$$
d N / d t=E_{t}[a] N\left(1-\frac{N}{C}\right)^{k}
$$

Hence, we have reduced inhomogeneous infinitely-dimensional logistic equation (2.11) to a single equation for $q(t)$,

$$
\frac{d q}{d t}=\left(1-\frac{N(0) M_{0}(q(t))}{C}\right)^{k}, q(0)=0 .
$$

The keystone variable $q(t)$ plays the role of "internal time" (as can be seen from equation (2.12)), such that the dynamics of inhomogeneous logistic model (2.11) with respect to the internal time is identical to the dynamics of the inhomogeneous Malthusian model with respect to the regular "external", or chronological time. The principle difference is that in general, $q(t)$ tends to $q^{*}<\infty$ as $t \rightarrow \infty$, which is a solution to the equation $M_{0}\left(q^{*}\right)=C / N(0)$.

Hence, the limit equilibrium state of inhomogeneous logistic model (2.11) will coincide with the current state of the inhomogeneous Malthus model at the instant $q^{*}$. The final stable population size and the final distribution of the parameter $a$ are, respectively

$$
\begin{gathered}
N^{*}=N(0) M\left(q^{*}\right), \\
P^{*}(a)=P_{0}(a) e^{q * a} / M_{0}\left(q^{*}\right) .
\end{gathered}
$$

Let us emphasize a notable property of the inhomogeneous logistic model (2.11) with a distributed Malthusian parameter: it remains inhomogeneous at any instant and has a non-trivial limit distribution of the parameter as $t \rightarrow \infty$. Every clone that was present initially will be present in the limit stable state. Therefore, inhomogeneous logistic model illustrates the phenomenon of "survival of everybody" in the population, in contrast to Darwinian "survival of the fittest".

\section{Selection systems and the Reduction theorem}

In Section 2 we have seen the effectiveness of the HKV method for expanding and enriching the analysis by introducing heterogeneity to some simple population models. It motivates us to develop the method in application to more general systems. 


\subsection{Selection systems}

A selection system is a mathematical model of an inhomogeneous population, in which every individual is characterized by a vector-parameter $\mathbf{a}=\left(a_{1}, \ldots, a_{n}\right)$ that takes on values from some set $A$. The parameter a specifies an individual's inherited invariant properties and does not change with time; just as before, the set of all individuals with a given value of the vector-parameter $\mathbf{a}$ in the population is called a-clone. Some general theorems for selection systems ("systems with inheritance") were proved in $[13,14]$.

Let $l(t, \mathbf{a})$ be the density of the population at time $t$ over the parameter a, so that the total population size is given by $N(t)=\int_{A} l(t, \mathbf{a}) d \mathbf{a}$, and the current population distribution is $P(t, \mathbf{a})=l(t, \mathbf{a}) / N(t)$. Denote $F(t, \mathbf{a})$ to be the per capita reproduction rate at time $t$. We assume here that the reproduction rate of every a-clone does not depend on other individual clones separately but can depend on the actual value of a and on the "environment" at $t$ moment. Most of the population characteristics, which we account for in the model, are functions of some averages over the populations, which are referred to here "regulators". Formally, a regulator has the form $G(t)=\int_{A} g(\mathbf{a}) l(t, \mathbf{a}) d \mathbf{a}$, where $g(\mathbf{a})$ is an appropriate function. The total population size is the most important regulator, corresponding to $g(\mathbf{a}) \equiv 1$. In many cases the reproduction rate may depend on mean values over the population distribution of the form $E_{t}[g]=\int_{A} g(\mathbf{a}) P(t, \mathbf{a}) d \mathbf{a}$. Let us emphasize that these mean values can be computed with the help of corresponding regulators according to the formula $E_{t}[g]=G(t) / N(t)$.

Overall, we specify for each model a finite set of regulators $\mathbf{G}(t)=\left\{G_{1}(t), \ldots G_{m}(t)\right\}$, which contains the total population size; we assume that the individual reproduction rate can depend on this set of regulators at each time moment. Then the population dynamics can be described by the following master model of a selection system:

$$
\begin{gathered}
\frac{d l(t, \mathbf{a})}{d t}=l(t, \mathbf{a}) F(t, \mathbf{a}), \\
F(t, \mathbf{a})=\sum_{i=1}^{n} u_{i}(t, \mathbf{G}) \varphi_{i}(\mathbf{a}),
\end{gathered}
$$

where $u_{i}(t, \mathbf{G})$ are continuous functions. The initial distribution $P(0, \mathbf{a})$ and the initial population size $N(0)$ are assumed to be known.

The mathematical form of the reproduction rate suggests (from a biological point of view) that the individual's fitness depends on a given finite set of traits. The function $\varphi_{i}(\mathbf{a})$ in $(3.2)$ may describe quantitative contribution of a particular $i$-th trait to the total fitness. The function $u_{i}(t, \mathbf{G})$ describes the relative importance (weight) of the $i$-th trait contribution, which at every time moment can depend on the state of the environment, population size, the mean, variance, covariance, and other statistical characteristics of the traits.

The current probability distribution solves the replicator equation:

$$
\frac{d P(t, \mathbf{a})}{d t}=P(t, \mathbf{a})\left(F(t, \mathbf{a})-E_{t}[F]\right)
$$

Replicator equations are among the basic tools in mathematical theory of selection and evolution, see, e.g., [15]. Some detailed examples of the selection system (3.1)-(3.2) and solutions of corresponding replicator equation will be given in the subsequent sections.

\subsection{Reduction theorem}

In model (3.1) the regulators and hence the reproduction rate $F(t, \mathbf{a})$ are not given explicitly but should be computed using the current pdf $P(t, \mathbf{a})$ at each time moment, so the model in the general case is a nonlinear equation of infinite dimensionality. Nevertheless, it can be reduced to a Cauchy problem for 
the escort system of ODE of dimensionality equal to the number of traits. To this end, introduce the generating functional:

$$
\Phi(r ; \lambda)=\int_{A} r(\mathbf{a}) \exp \left(\sum_{i=1}^{n} \lambda_{i} \varphi_{i}(\mathbf{a})\right) P(0, \mathbf{a}) d \mathbf{a}
$$

where $\lambda=\left(\lambda_{1}, \ldots \lambda_{n}\right)$ and $r(\mathbf{a})$ is a measurable function on $A$.

Define auxiliary (keystone) variables as a solution to the escort system of differential equations:

$$
\frac{d q_{i}(t)}{d t}=u_{i}\left(t, \mathbf{G}^{*}(t)\right), q_{i}(0)=0, i=1, \ldots n,
$$

where

$$
\mathbf{G}^{*}(t)=\left\{G_{1}^{*}(t), \ldots G_{m}^{*}(t)\right\}
$$

and

$$
G_{k}^{*}(t)=N(0) \Phi\left(g_{k}, \mathbf{q}(t)\right), \mathbf{q}(t)=\left\{q_{1}(t), \ldots q_{n}(t)\right\}
$$

Denote

$$
K_{t}(\mathbf{a})=e^{\sum_{i=1}^{n} q_{i}(t) \varphi_{i}(\mathbf{a})} .
$$

Reduction Theorem [27]. Let $0<T \leq \infty$ be the maximal value of $t$ such that Cauchy problem (3.5) has a unique solution $\{\mathbf{q}(t)\}$ at $t \in[0, T)$. Then the functions:

$$
\begin{gathered}
l(t, \mathbf{a})=l(0, \mathbf{a}) K_{t}(\mathbf{a}) \\
G_{k}(t)=G_{k}^{*}(t)=N(0) \Phi\left(g_{k}, \mathbf{q}(t)\right),
\end{gathered}
$$

satisfy System (3.1)-(3.2) at $t \in[0, T)$.

Specifically, the total size of the population

$$
N(t)=N(0) \Phi(1, \mathbf{q}(t))=N(0) E_{0}\left[K_{t}\right] .
$$

As a corollary, we obtain the central formula for the current distribution of the system:

$$
P(t, \mathbf{a})=P(0, \mathbf{a}) K_{t}(\mathbf{a}) / E_{0}\left[K_{t}\right] .
$$

The Reduction Theorem applies the powerful HKV (Hidden Keystone Variables) method for solving selection systems (3.1)-(3.2) and corresponding replicator equations (3.3). Introducing auxiliary variables, which solve the escort system of non-autonomous differential equations (3.5) (see also (3.11) below), allows us to write down the solution of a selection system and the corresponding replicator equation and to find all its statistical characteristics of interest.

The general HKV method is simplified in an important case of the reproduction rate

$$
F(t, \mathbf{a})=\sum_{i=1}^{n} u_{i}(t, \mathbf{G}) \varphi_{i}(\mathbf{a})
$$

with the regulators of the form $N(t), G_{i}(t)=N(t) E_{t}\left[\varphi_{i}\right]$ only. In this case, we can use the moment generating function (mgf) of the joint initial distribution of the variables $\varphi_{i}$,

$$
M_{0}(\lambda)=E^{0}\left[\exp \left(\sum_{i=1}^{n} \lambda_{i} \varphi_{i}\right)\right]
$$

instead of general functional (3.4). The resulting escort system reads 


$$
\frac{d q_{i}}{d t}=u_{i}(t, \mathbf{G}(t)), q_{i}(0)=0, i=1, \ldots n,
$$

where $G_{i}(t)$ are defined via formulas

$$
\begin{aligned}
& N(t)=N(0) M_{0}(\mathbf{q}(t)), \\
& E_{t}\left[\varphi_{i}\right]=\partial_{i} \ln M_{0}(\mathbf{q}(t)) .
\end{aligned}
$$

The solution to the corresponding replicator equation is

$$
P(t, \mathbf{a})=P(0, \mathbf{a}) K_{t}(\mathbf{a}) / E_{0}\left[K_{t}\right]
$$

where $E_{0}\left[K_{t}\right]=M_{0}(\mathbf{q}(t))$.

In the following sections we demonstrate the HKV method at work.

\section{Inhomogeneous population models}

\subsection{Global demography}

The growth of the world population up to $\sim 1990$ was described with high accuracy by the hyperbolic law $[9]$ :

$$
N(t)=\frac{C}{T-t}
$$

with $C \approx 2 * 10^{11}, T \cong 2025$.

Formula (4.1) describes the hyper-exponential growth of humankind. This formula solves the quadratic growth model

$$
\frac{d N}{d t}=N^{2} / C
$$

in which the growth rate is proportional to the number of pair contacts in the whole of humankind and the individual reproduction coefficient is proportional to the total world population. This fact is difficult to interpret from the point of view of elementary processes and it seems evidently wrong even for small populations. In addition, equations (4.1), (4.2) predict a non-realistic demographic explosion at some point in time as we approach the year $T \cong 2025$. This means that $N(t) \rightarrow \infty$ when $t \rightarrow T$, and the same is true for the population growth rate and individual reproduction coefficient.

Let us note that, usually, Eqs. (4.1) and (4.2) are considered to be equivalent; this assertion was the basis for phenomenological theory of global demography developed in $[19,20]$. In this theory, the underlying assumption of the humankind uniformity and the interpretation of the "quadratic law" (4.2) in terms of the information community hypothesis are, perhaps, unrealistic, especially when dealing with prehistoric human populations that were composed of comparatively small low-interbreeding subgroups. Let us emphasize that, paradoxically (from the standpoint of the theory developed in [20]), the hyperbolic growth was observed in the past but has substantially slowed down during the last decades when most of the world population indeed became an "information community".

The mathematical part of the theory [19] was based on some modification of the quadratic equation (4.2), which was considered as an exceptional phenomenon inhered to humankind only.

In our opinion, equation (4.2) cannot be the starting point of any realistic demographic theory because it apparently makes no biological sense, at least, for large populations. Then, the problem arises: why the unacceptable quadratic equation (4.2) implies a good fit of empirical data (4.1)? The answer is: the hyperbola $N(t)=\frac{C}{T-t}$ is implied not only by the quadratic growth model but also by more plausible Malthusian inhomogeneous model (2.9) with exponential initial distribution (see [22] for details). 
Given that any real population is inhomogeneous, the simplest inhomogeneous Malthusian model is more acceptable as a starting point for global demography modeling then the quadratic growth model. The population increases in such a way that the distribution of the reproduction rate is exponential at every instant $t<T$ with the mean $E_{t}[a]=1 /(T-t)$.

The "demographic explosion" occurs at the moment $t=T$ when not only $N(T)=\infty$, but also $E_{T}[a]=\infty$ and $\operatorname{Var}_{T}[a]=\infty$. It is a corollary of the obviously wrong assumption (incorporated implicitly into quadratic growth model) that the individual reproduction rate may take arbitrary large values with nonzero probability. Hence, a natural way to eliminate the unrealistic "demographic explosion" from the model is to take into account that possible values of the reproduction rate should be limited. When the reproduction rate $a$ in model (4.1) is bounded, i.e. the rate has an exponential distribution truncated in the interval $[0, c)$ (specifically for real demographic data, $c \approx 0.114[22]$ ) then one can show that

$$
N(t)=N(0) \frac{T}{T-t}\left(1-e^{-c(T-t)}\right) .
$$

It follows from (4.3) that $N(t)$ is finite, even though indefinitely increasing, for all $t$.

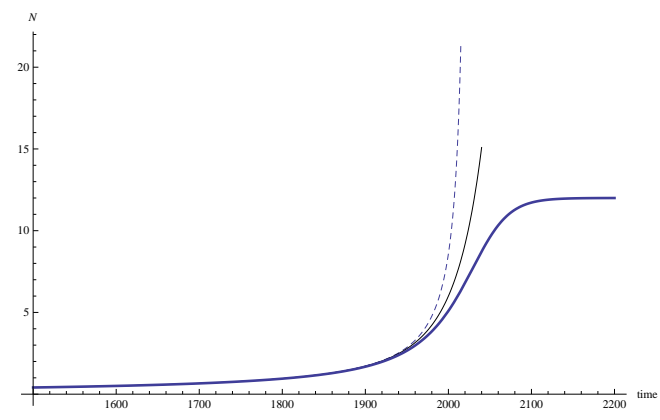

Figure 1. World population size, $N$ (in billions), dependently on time $t: 1$ (dashed) model (3.1) with exponentially distributed rate; 2 - model (4.3); 3 (thick) - inhomogeneous logistic model (3.7) with $C=12$ and $k=2$.

The next step toward more realistic demographic model is the inhomogeneous logistic equation

$$
\frac{d l(t ; a)}{d t}=a l(t ; a)\left(1-\frac{N}{C}\right)^{k}
$$

where $a$ is the distributed growth rate, $C$ is the total carrying capacity, and $k>0$ is a constant. This equation was studied in Section 2. Figure 1 shows that the solutions of all considered models are very close to the hyperbola for a long time (up to 1990 at corresponding values of coefficients), but dramatically differ at large $t$.

The last model shows a transition from prolonged hyperbolical growth (the phase of "hyper-exponential" development) to the brief transitional phase of "nearly exponential" growth (the phase of "demographic transition") accomplished by a sharp increase of the variance of the reproduction rate to a stabilized regime, see Figure 2. It means that according to the last model humankind stay inhomogeneous forever; see discussion in s. 3.4. We may conclude that the hyperbolic growth of the humankind for a long time period was not an exclusive phenomenon but obeyed the same laws as any heterogeneous biological population.

\subsection{Models of population extinction}

The problem of population extinction is one of the most important problems in modern ecology. The developed HKV method can be successfully applied to this problem. In particular, different peculiarities 


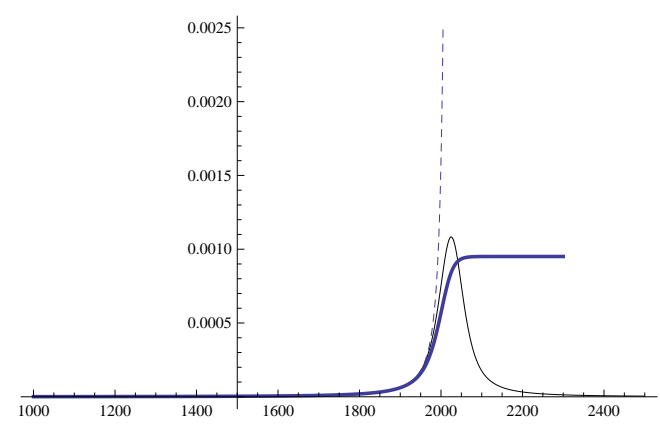

FiguRE 2. Variances $\operatorname{Var}_{t}(a)$ of the rate $a$ dependently on time $t$ for the models 1-3 (see Legend for Figure 1).

of the dynamics of population dying may be explained by the population heterogeneity. Let us consider the inhomogeneous Malthusian extinction model

$$
\frac{d l(t, a)}{d t}=-a c l(t, a), N(t)=\int_{A} l(t, a) d a .
$$

where $c$ is a scaling constant. Then

$$
N(t)=N(0) \int_{A} e^{-a c t} P(0, a) d a=N(0) L(c t),
$$

where $L$ is the Laplace transform of the initial distribution $P(0, a)$. The theorem of S. Bernstein ([7], v.2, ch.X111, 4) states, that a function $L$ is the Laplace transform of a pdf if and only if $L$ is completely monotone and $L(0)=1$. Hence, the population size $N$ in inhomogeneous Malthusian extinction model (4.4) can change as an arbitrary completely monotone function on time, dependently on the initial distribution.

As an example, let us consider one of the oldest and important problems in forest ecology, the problem of tree stand self-thinning. A number of tree interactions, variations in genetic structure, and various environmental conditions affect the growth and death of trees in complex ways. It seems to be impossible to take into account all factors that impact death rates of trees in explicit form within the frameworks of a unit model.

A promising way to overcome these difficulties is to construct tree population models with distributed values of the mortality rate $a$. It was shown in $[21,23]$ that different formulas of forest stand self-thinning can be considered as solutions of inhomogeneous Malthusian extinction model (4.4). Let us consider, for example, a known formula suggested by Khilmi [34] for tree number $N(t)$ of even-age tree stand,

$$
N(t)=N(0) \exp \left(a_{0}\left(e^{-c t}-1\right)\right) .
$$

One can notice that this formula coincides with a well-known Gompertz function. It was shown in [21] that the last formula is an exact solution of (4.4) if the mortality rate $a$ has the Poisson distribution with average $a_{0}$ at the initial time point. In this case, the distribution of $a$ at any moment of time is also Poisson with the mean $E_{t}[a]=a_{0} e^{-c t}$. Indeed, the mgf of the Poisson distribution $M(\lambda)=\exp \left(a_{0}\left(e^{-c t}-1\right)\right)$, and all assertions follow from formulas of s.2.

Thus, the Hilmi formula describes the Malthusian process of decreasing of the inhomogeneous population size; the population is divided into countable number of disjoint groups such that individuals from $i$-th group, $i=0,1,2, \ldots$ have the mortality rate $i c$ and the initial size of the group is $l_{0}(i)=N(0) \exp \left(-a_{0}\right) a_{0}{ }^{i} / i$ !

It is difficult to give an acceptable interpretation of this model with infinite number of groups. Individuals from groups with large $i$ have unrealistic large extinction rate and will be eliminated rapidly from the 
population; more of that, the initial size of such a group may be less than 1 with large $i$. Thus, it is reasonable to consider the subdivision of a tree population only to finite number of groups with different extinction rates that corresponds to the truncated Poisson distribution.

Let parameter $a$ can assume only finite number of values $i=0,1, \ldots k$. Then for all $t$ the distribution of the parameter $a$ is again the $k$-truncated Poisson distribution and the population size is

$$
N(t)=N(0) c(k) \sum_{i=0}^{k}\left(\exp \left(-a_{0}\right) a_{0}\right)^{i} / i ! .
$$

This formula is biologically more meaningful than the initial Khilmi formula with $k=\infty$; it allows to fit real data more precisely. Moreover, it is now possible to choose an unknown number $k$ of different $a$-groups by best fitting, for different $k$, to the observed time series of the population sizes. Thus we can estimate the number of groups of trees having different "surviving levels". For example, computations for pine tree stands give an estimate of $k=7-10$. The accuracy of data fitting is better than $5 \%$ of mean-square deviation (see $[21,23]$ for more details).

Some other self-thinning formulas, such as the power law can be deduced and then modified by the same approach $[21,23]$.

\subsection{Inhomogeneous Ricker equation}

Let us now demonstrate how to solve the inhomogeneous population models, which depend on a vector parameter a. As an example, let us consider a version of the well-known Ricker equation, which depends on two distributed parameters, $a_{1}=\beta, a_{2}=\mu$ :

$$
\frac{d l(t ; \beta, \mu)}{d t}=l(t ; \beta, \mu)\left(\beta e^{-c N(t)}-\mu\right)
$$

where $c$ is a scale constant.

Let $M\left(\lambda_{1}, \lambda_{2}\right)$ be the mgf of the joint initial distribution of $\beta$ and $\mu$. Then the escort system reads

$$
\frac{d q_{1}}{d t}=e^{-c N(0) M\left(q_{1},-t\right)}, q_{1}(0)=0
$$

$\frac{d q_{2}}{d t}=-1, q_{2}(0)=0$, hence $q_{2}(t)=-t$.

Equation (4.7) can be solved for known mgf $M\left(\lambda_{1}, \lambda_{2}\right)$ and then the solution to $(4.6)$ is $l(t ; \beta, \mu)=$ $l(0 ; \beta, \mu) \exp \left(\beta q_{1}(t)-\mu t\right)$; the total population size and the parameters distribution are given by the formulas

$$
\begin{gathered}
N(t)=N(0) M\left(q_{1}(t),-t\right), \\
P(t ; \beta, \mu)=P(0 ; \beta, \mu) e^{\left(\beta q_{1}(t)-\mu t\right)} / M\left(q_{1}(t),-t\right) .
\end{gathered}
$$

For example, let the parameters $\beta$ and $\mu$ be independent and exponentially distributed in $[0, \infty)$ with the means $s_{1}$ and $s_{2}$ at the initial instant. Then $M(q,-t)=\frac{s_{1} s_{2}}{\left(s_{1}-q\right)\left(s_{2}+t\right)}$, and equation (4.7) for the auxiliary variable reads $\frac{d q_{1}}{d t}=e^{-c N(0) \frac{s_{1} s_{2}}{\left(s_{1}-q_{1}\right)\left(s_{2}+t\right)}}$.

This equation has a stable state at $q_{1}=s_{1}$. As $t \rightarrow \infty, q_{1}(t) \rightarrow s_{1}$ and $M\left(q_{1},-t\right) \rightarrow \infty$; so the total population size tends to infinity and the population density concentrates at the value $\mu=0$ of the parameter $\mu$ and vanishes in any finite interval of values of the parameter $\beta$. This unrealistic behavior is a corollary of two assumptions: the death rate $\mu$ can be arbitrary small and the growth coefficient $\beta$ can be arbitrary large. It can be proven that if the parameters $\beta$ and $\mu$ are independent and there exist $\max \beta=\beta^{*}<\infty$ and $\min \mu=\mu^{*}>0$, then the limit distribution of the parameters will be concentrated in the point $\left(\beta^{*}, \mu^{*}\right)$. 


\subsection{The principle of limiting factors in modeling of early biological evolution}

Principle of limiting factors, according to Liebig [39] (see also [48] for mathematical formulation) states that at any given moment the rate of a process is determined by the factor whose sufficiently small modification produces a change of the rate; it is assumed that similar changes in other factors do not affect the rate. The principle of limiting factors was actually used in a model of early biological evolution suggested in [53]. Each organism was characterized by the vector a where the component $a_{i}$ is the thermodynamic probability that protein $i$ is in its native conformation. In order to study the connection between molecular evolution and population, the authors suppose that the organism death rate $d$ depends on the stability of its proteins as $d=d_{0}\left(1-\min a_{i}\right), d_{0}=b /\left(1-a_{0}\right), b$ is the birth rate, $a_{0}$ is the native state probability of a protein. Hence, neglecting possible mutations (accounted for by the authors in their simulations), the model can be formalized as the system

$$
\left.\frac{d l(t, a)}{d t}=l(t, a) d_{0}\left(m(a)-a_{0}\right)\right)
$$

where $m(a)=\min \left[a_{1}, \ldots a_{n}\right]$. In what follows we let $d_{0}=1$ for simplicity. It was assumed in [53] that the values $a_{i}$ are independent and have the Boltzmann distribution. We can consider $a_{i}$ as the $i$-th realization of a random variable with a common pdf $f(a)$.

Let $G(a)=\int_{0}^{a} f(x) d x$ be the cumulative distribution function. Then, it is well known that the pdf of $\min \left[a_{1}, \ldots a_{n}\right]$ is equal to $g(a)=n(1-G(a))^{n-1} f(a)$. Equation (4.8) is a version of the inhomogeneous Malthusian equation, which can now be solved explicitly at any given pdf $f(a)$. In particular, if

$$
f(a)=\exp (-a / T) / Z, Z=\sum_{a} \exp (-a / T)
$$

is the Boltzmann distribution with $a>0$, then

$$
\left.g(a)=n(1-G(a))^{n-1} f(a)=\left(n\left(\sum_{x>a} \exp (-x / T)\right)^{n-1}\right) \exp (-a / T) / \sum_{x} \exp (-x / T)\right) .
$$

For distribution (4.9) with continuous range of values of $a, a \in(0, \infty), Z=T, 1-G(x)=\exp (-x / T)$ and

$$
g(a)=n(\exp (-a(n-1) / T) \exp (-a / T) / T=n / T \exp (-a n / T) .
$$

If $a \in(0, E)$, then $Z=T(1-\exp (-E / T)), 1-G(a)=\frac{\exp ((E-a) / T)-1}{\exp (E / T)-1}$, and

$$
g(a)=\frac{n \exp (-a / T)}{T(1-\exp (-E / T))}\left[\frac{1-\exp ((E-a) / T)}{1-\exp (E / T)}\right]^{n-1} .
$$

Let $M_{0}(\lambda)=E_{0}[\exp (\lambda m)]$. For initial distribution $(4.10), M_{0}(\lambda)=\frac{1}{1-\lambda T / n}$. Hence,

$$
\begin{gathered}
l(t, a)=l(0, a) \exp \left(\left(m(a)-a_{0}\right) t\right), \\
N(t)=N(0) \exp \left(-a_{0} t\right) \frac{1}{1-t T / n}, \\
P(t, a)=P(0, a) \exp (m(a) t)(1-t T / n) .
\end{gathered}
$$

At the moment $t_{\max }=n / T$ the population "blows up": $N(t)$ and $l(t, a)$ tend to infinity as $t \rightarrow t_{\max }$. Let us denote $p(t, a)=P(t,\{a: m(a)=a\})$. Then at $t<t_{\max }$

$$
p(t, a)=n / T \exp (-a n / T+a t)(1-t T / n)=(n / T-t) \exp (a(t-n / T)) .
$$

The probability $P(t,\{a: m(a)<a\})$ tends to 0 for any finite $a$ as $t \rightarrow t_{\max }$. Loosely speaking, the total "probability mass" goes to infinity after a finite time interval. So, we should conclude that model (4.8), 
(4.9), which allow arbitrary large values of the parameter $a$ with nonzero probability have no "physical" sense.

This problem can be eliminated by taking the initial distribution, which allows only bounded values of the parameter $a$. For pdf (4.11), the integral

$$
M_{0}(\lambda)=\int_{0}^{E} e^{\lambda x} g(x) d x
$$

is finite for any $\lambda$; although it cannot be expressed in quadratures we can obtain much information about the system distribution and its dynamics. The current pdf is

$$
p(t, a)=\frac{n}{T(\exp (E / T)-1)^{n}} \exp ((E-a) / T)(\exp ((E-a) / T)-1)^{n-1} \frac{e^{a t}}{M_{0}(t)},
$$

where $M_{0}(t)$ is finite for all $t$. So, the pdf is defined and finite at any time moment, in contrast to the previous case. The total distribution concentrates with time at the point $a=E$, which provides the maximal reproduction rate. Let us emphasize that the pdf $p(t, a)$ does not depend on the native state probability $a_{0}$.

\section{Discrete time selection systems}

\subsection{Reduction theorem for maps}

A similar theory can be developed for selection systems and replicator equations with discrete time [26]. Once again, let $l(t, a)$ be the population density at moment $t$; the discrete-time version of the model (3.1) of selection systems reads

$$
\begin{gathered}
l(t+1, a)=l(t, a) w_{t}(a), \\
w_{t}(a)=\exp \left(\sum_{i=1}^{n} u_{i}(t, G(t)) \varphi_{i}(a),\right.
\end{gathered}
$$

where again $G(t)=G_{1}(t), \ldots G_{m}(t), G_{i}(t)=N(t) E_{t}\left[g_{i}\right], N(t)$ is the total population size and $u_{i}(t, G)$ are continuous functions.

Define the current pdf $P_{t}(a)=l(t, a) / N(t)$; it solves the discrete-time replicator equation

$$
P(t+1, a)=P(t, a) w_{t}(a) / E_{t}\left[w_{t}\right] .
$$

Let us formulate the reduction theorem for selection system (5.1). Denote $U_{i}(t)=\sum_{j=0}^{t} u_{i}(j, G(j))$, and

$$
W(t, a)=\prod_{k=0}^{t} w_{k}(a)=\exp \left(\sum_{i=1}^{n} U_{i}(t) \varphi_{i}(a)\right) .
$$

Consider the vector-function $\mathbf{U}(\mathbf{t})=\left(\mathbf{U}_{\mathbf{1}}(\mathbf{t}), \ldots \mathbf{U}_{\mathbf{n}}(\mathbf{t})\right)$. Suppose that the initial distribution $P(0, a)$ is given and $\Phi(r ; \lambda)$ is the generating functional (3.4).

Theorem 4. The total population size, the regulators and the system distribution can be computed recurrently with the help of the following system:

$$
\begin{gathered}
N(t)=N(0) \Phi(1 ; \mathbf{U}(\mathbf{t}-\mathbf{1})) ; \\
G_{i}(t)=N_{0} \Phi\left(g_{i} ; \mathbf{U}(\mathbf{t}-\mathbf{1})\right) ; \\
P(t, a)=P(0, a) W(t-1, a) / E_{0}[W(t-1, a)]
\end{gathered}
$$

Theorem 4 reduces model (5.1)-(5.2) (which in general is infinitely dimensional) to a finite system of recursive equations. Formulas (5.3)-(5.4) give the solution to replicator equation (5.2). 


\subsection{Inhomogeneous logistic map}

A well-known logistic map is of the form $N_{t+1}=\lambda N_{t}\left(1-N_{t}\right), 0<\lambda<4$ and $0 \leq N_{t} \leq 1$. The following inhomogeneous version of this map was studied in $[26]$ :

$$
l(t+1, a)=\lambda a l(t, a)\left(1-N_{t}\right)
$$

where $a$ is the distributed parameter, $0<a<1$. Denote $w_{t}(a)=\lambda a\left(1-N_{t}\right)$.

Let $W(t, a)=\prod_{k=0}^{t} w_{k}(a)=\lambda^{t+1} \exp ((t+1) \ln a+U(t))$,

where $U(t)=\sum_{k=1}^{t} \ln \left(1-N_{k}\right)$. Then, according to (5.4) the current pdf

$$
P(t, a)=P(0, a) W(t-1, a) / E_{0}[W(t-1, a)]=P(0, a) a^{t} / E_{0}\left[a^{t}\right] .
$$

The inhomogeneous logistic model is reduced to the system

$$
\begin{gathered}
N_{t+1}=E_{t}[w] N_{t}=\lambda E_{t}[a] N_{t}\left(1-N_{t}\right), \\
E_{t}[a]=E_{0}\left[a^{t+1}\right] / E_{0}\left[a^{t}\right] .
\end{gathered}
$$

The current distribution (5.5) of the model has a simple form. In contrast, the behavior of trajectories may be very complex. For example, let $P_{0}(a)$ be the Beta-distribution in $[0,1]$ with parameters $\alpha, \beta$. Then $E_{t}[a]=(t+\alpha) /(t+\alpha+\beta)$. Choosing an appropriate value of $0<\lambda<4$, we can observe (as $\left.t \rightarrow \infty\right)$ any possible behavior of the plain logistic model as the final dynamical behaviors of the inhomogeneous logistic model. In particular, at $\lambda=4$ almost all cycles of Feigenbaum's cascade appear in the course of time and realize as parts of a single trajectory, as a result of the "inner" bifurcations of the inhomogeneous logistic model. The trajectory $\left\{N_{t}\right\}_{0}^{\infty}$ mimics the bifurcation diagram of the plain logistic map. Figure 3 illustrates this assertion.

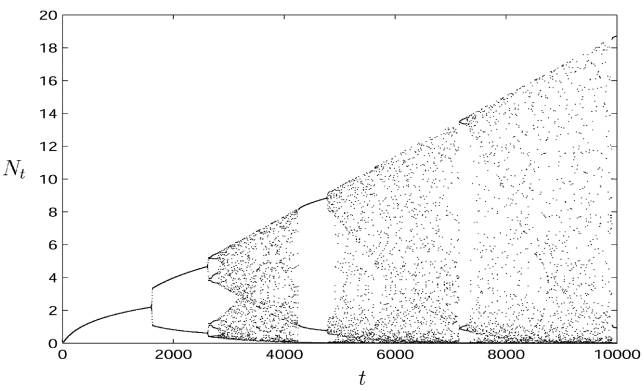

FIgURE 3. The trajectory of total population size for inhomogeneous logistic model with Beta-distributed parameter $a\left(\lambda=4, E_{0}[a]=0.1, \operatorname{Var}_{0}[a]=0.005\right)$.

\subsection{Selection in Natural Rotifer Community}

The mathematical model of zooplankton populations under toxicant exposure was suggested in [49] and studied systematically in [3]. The model depends on the parameters $a$, characterizing the environment quality, and $\gamma$, which is the species-specific parameter. The model was given by the equation

$$
N_{t+1}=N_{t} \exp \left(-a+\frac{1}{N_{t}}-\frac{\gamma}{N_{t}^{2}}\right) .
$$

Let us consider the model of a community that consists of different rotifer populations (see [25]); individuals within the population may have different reproduction capacities under constant toxicant exposure. The model is of the form (5.1), $l(t+1, a)=l(t, a) w_{t}(a)$, where $\mathbf{a}=(a, \gamma)$ and $w_{t}(a, \gamma)=\exp \left(-a+\frac{1}{N_{t}}-\frac{\gamma}{N_{t}^{2}}\right)$. 
Then

$$
W(t ; a, \gamma)=\exp \left(-(t+1) a+\sum_{k=0}^{t} 1 / N_{k}-\gamma \sum_{k=0}^{t} 1 / N_{k}^{2}\right) .
$$

Let $M\left(\lambda_{1}, \lambda_{2}\right)$ be the mgf of the initial distribution of $a$ and $\gamma$. Then

$$
\begin{gathered}
E_{0}[W(t ; a, \gamma)]=\exp \left(\sum_{k=0}^{t} 1 / N_{k}\right) M\left(-(t+1),-\sum_{k=0}^{t} 1 / N_{k}^{2}\right), \\
N_{t}=N_{0} \exp \left(\sum_{k=0}^{t-1} 1 / N_{k}\right) M\left(-t,-\sum_{k=0}^{t-1} 1 / N_{k}^{2}\right) \\
P(t ; a, \gamma)=P(0 ; a, \gamma) \exp \left(-t a-\gamma \sum_{k=0}^{t-1} 1 / N_{k}^{2}\right) / M\left(-t,-\sum_{k=0}^{t-1} 1 / N_{k}^{2}\right) .
\end{gathered}
$$

These formulas completely solve the inhomogeneous model of rotifer community.

Notice that inhomogeneous logistic and Ricker' maps show transitions from deterministic to chaotic behaviors; in contrast, the model of rotifer community shows the transition from almost chaotic to deterministic behavior. The trajectory $N_{t}$ may have a very complex transition regime from the initial to the final behavior; see Figure 4.

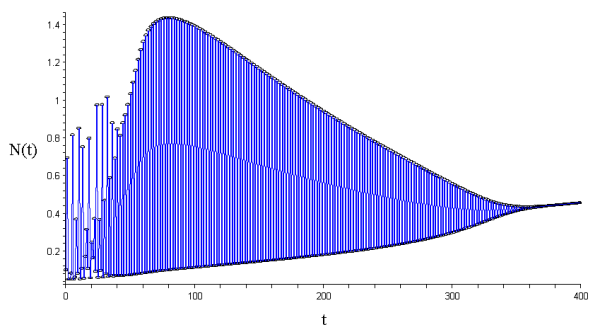

FiguRE 4. The trajectory of total population size for inhomogeneous rotifer' model where independent parameters $a, \gamma$ are both $\Gamma$ - distributed; $E_{0}[a]=4, \operatorname{Var}_{0}[a]=0.01$; $E_{0}[\gamma]=0.06, \operatorname{Var}_{0}[\gamma]=0.03$.

\section{Inhomogeneous models of communities}

\subsection{Reduction Theorem}

Consider the model of a community consisting of $r$ interacting populations. We assume once again that every individual is characterized by their own value of vector-parameter $a$. Let $l^{j}(t, a)$ be the density of $j$-th population at moment $t$. In this section we consider the model of an inhomogeneous community where the reproduction rates can depend on current characteristics of every population in the community composing a "regulator". Formally, we consider the set of $m$ regulators, each of which is the $r$-dimension vector-function $G_{i}(t)=\left(G_{i}^{1}(t), \ldots G_{i}^{r}(t)\right), i=1, \ldots n$ where:

$$
G_{i}^{j}(t)=\int_{A} g_{i}(a) l^{j}(t, a) d a
$$

Each regulator corresponds to appropriate weight function $g_{i}$. A finite set of the regulators corresponds to each specific model; we denote this set as $\mathbf{G}(\mathbf{t})=\left(\mathbf{G}_{\mathbf{1}}(\mathbf{t}), \ldots \mathbf{G}_{\mathbf{n}}(\mathbf{t})\right)$. 
The current population sizes $N^{j}(t)=\int_{A} l^{j}(t, a) d a$ compose the regulator of a special importance, $N(t)=\left(N^{1}(t), \ldots N^{r}(t)\right)$. We assume that $N(t)$ is included in the set of the model's regulators. The distribution of $j$-th population in the community is by definition $P^{j}(t, a)=l^{j}(t, a) / N^{j}(t)$.

The model of inhomogeneous community considered here (see [28]) is of the form:

$$
\begin{aligned}
& d l^{j}(t, a) / d t=l^{j}(t, a) F^{j}(t, a) \\
& F^{j}(t, a)=\sum_{i=1}^{n} u_{i}^{j}(t, \mathbf{G}) \varphi_{i}(a),
\end{aligned}
$$

where the functions $u_{i}^{j}$ can be specific for each trait and each population. The initial pdf-s $P^{j}(0, a)$ and the initial population sizes $N^{j}(0)$ are assumed to be given. The current pdf $P^{j}(t, a)$ solves the replicator equation:

$$
d P^{j}(t, a) / d t=P^{j}(t, a)\left(F^{j}(t, a)-E_{t}^{j}\left[F^{j}\right]\right)
$$

The theory for inhomogeneous community model (6.1)-(6.4) is similar to the theory presented in Section 3 for inhomogeneous populations up to more complex technical details. Theorem 5 below reduces complex model (6.1)-(6.3) to an escort system of ordinary non-autonomous equations of dimension $r \times n$ and gives the solution to replicator equation (6.4).

Let us introduce the keystone variables as the solution to the following Cauchy problem:

$$
d q_{i}^{j} / d t=u_{i}^{j}\left(t, G_{i}^{*}(t)\right), \quad q_{i}^{j}(0)=0, \quad j=1, \ldots r, \quad i=1, \ldots n .
$$

Here:

$$
q^{j}(t)=\left(q_{1}^{j}(t), \ldots q_{n}^{j}(t)\right), G_{i}^{* j}(t)=N^{j}(0) \Phi^{j}\left(g_{i} ; q^{j}(t)\right),
$$

where $\Phi^{j}(r ; \lambda)$ is the generating functional (3.8) for the initial distribution $P^{j}(0, a)$; in particular,

$$
N^{* j}(t)=N^{j}(0) \Phi^{j}\left(1 ; q^{j}(t)\right) .
$$

Denote

$$
K_{t}^{j}(a)=\exp \left(\sum_{i=1}^{n} q_{i}^{j}(t) \varphi_{i}(a)\right)
$$

Theorem 5 [28]. Assume that Cauchy problem (6.5) has a unique solution at $t \in[0, T), 0<T \leq \infty$. Then the functions:

$$
\begin{gathered}
l^{j}(t, a)=l^{j}(0, a) K_{t}^{j}(a) \\
G_{i}^{j}(t)=N^{j}(0) \Phi^{j}\left(g_{i} ; q^{j}(t)\right) \\
N^{j}(t)=N^{j}(0) \Phi^{j}\left(1 ; q^{j}(t)\right)
\end{gathered}
$$

satisfy system (6.1)- (6.3) at $t \in[0, T)$. The pdf:

$$
P^{j}(t, a)=P^{j}(0, a) K_{t}^{j}(a) / E_{0}\left[K_{t}^{j}\right]=P^{j}(0, a) K_{t}^{j}(a) / \Phi^{j}\left(1 ; q^{j}(t)\right)
$$

solves the replicator equations (6.4).

Let us apply the general theory to some classical models of biological communities consisting of interacting inhomogeneous populations. 


\subsection{Inhomogeneous prey-predator Volterra model}

The classical prey-predator Volterra model in its simplest form reads

$$
\begin{aligned}
& d x / d t=a_{1} x-a_{2} x y, \\
& d y / d t=-a_{3} y+a_{4} x y,
\end{aligned}
$$

where $x(t)$ and $y(t)$ denote prey and predator densities, $a_{1}$ is the reproduction rate of the prey population, $a_{2}$ is the per capita rate of the consumption of prey by the predators, $a_{3}$ is the death rate of the predator, and $a_{4} / a_{2}$ is the fraction of prey biomass, which is converted into predator biomass.

Prey-predator model (6.7) is a well-studied mathematical object. Nevertheless, if a biological community consists of many populations of preys which can be attacked by predator, or if there are many populations of predators in the community, then corresponding multi-dimensional model is a complex system which is difficult to study. Below we show how many-dimensional prey-predator model can be reduced to 2-dimensional escort system of non-autonomous differential equations.

Let us consider the inhomogeneous version of this classical model supposing that parameters $a_{1}, a_{2}$, and $a_{3}$ are distributed and the ratio $a_{4} / a_{2}$ is fixed (and hence could be chosen equal to 1 ). We also assume that the reproduction and death processes are specific for each subpopulation, while the consumption is driven by the interaction of the prey (predator) subpopulation with the entire predator (prey) population.

Let $x\left(t ; a_{1}, a_{2}\right), y\left(t ; a_{3}\right)$ be the densities of the prey and predator populations over parameters $a_{1}, a_{2}$ and $a_{3}$ correspondingly, and $X(t)=\int_{A} x\left(t ; a_{1}, a_{2}\right) d a_{1} d a_{2}, Y(t)=\int_{A} y\left(t ; a_{3}\right) d a_{3}$ be the total sizes of the prey and predator populations. The total rate of consumption is equal to $Y(t) \int_{A} a_{2} x\left(t ; a_{1}, a_{2}\right) d a_{1} d a_{2}=$ $Y(t) X(t) E_{t}^{1}\left[a_{2}\right]$. Assuming the "proportional distribution" of prey among the predators we can write the inhomogeneous version of Volterra model in the form

$$
\begin{aligned}
& d x\left(t ; a_{1}, a_{2}\right) / d t=x\left(t ; a_{1}, a_{2}\right)\left(a_{1}-a_{2} Y(t)\right), \\
& d y\left(t ; a_{3}\right) / d t=y\left(t ; a_{3}\right)\left(G(t)-a_{3}\right),
\end{aligned}
$$

where $G(t)=\int_{A} a_{2} x\left(t ; a_{1}, a_{2}\right) d a_{1} d a_{2}=X(t) E_{t}^{1}\left[a_{2}\right]$.

The initial population sizes and initial distributions $P^{1}\left(0 ; a_{1}, a_{2}\right), P^{2}\left(0 ; a_{2}\right)$ are assumed to be given. The problems of interest are: to find the dynamics of total prey and predator population sizes, $X(t)$ and $Y(t)$, and to find frequencies of each clone in the populations, or, equivalently, to find the pdf-s $P^{1}\left(t ; a_{1}, a_{2}\right), P^{2}\left(t ; a_{2}\right)$.

Theorem 5 provides a method for answering these questions; the principal step is reduction of the complex system (6.8) to the escort system of ODE for keystone variables. It is instructive to deduce the escort system and the main results informally to clarify the main idea of the method in application to community models.

It is natural to assume that the parameter $a_{3}$ is stochastically independent on the parameters $a_{1}, a_{2}$. Let $M^{1}\left(\lambda_{1}, \lambda_{2}\right)$ be the mgf of the initial joint distribution of the parameters $a_{1}, a_{2}$, and $M^{2}\left(\lambda_{3}\right)$ be the mgf of the initial distribution of the parameter $a_{3}$.

Introduce the auxiliary keystone variables $q_{1}(t), q_{2}(t)$ as a solution to the Cauchy problem

$$
\begin{gathered}
d q_{1} / d t=Y(t), \\
d q_{2} / d t=G(t)=X(t) E_{t}^{1}\left[a_{2}\right], \\
q_{1}(0)=q_{2}(0)=0 .
\end{gathered}
$$

Then system (6.8) can be re-written formally as

$$
\begin{gathered}
d x\left(t ; a_{1}, a_{2}\right) / d t=x\left(t ; a_{1}, a_{2}\right)\left(a_{1}-a_{2} d q_{1} / d t\right), \\
d y\left(t ; a_{3}\right) / d t=y\left(t ; a_{3}\right)\left(d q_{2} / d t-a_{3}\right) .
\end{gathered}
$$

Its solution is

$$
x\left(t ; a_{1}, a_{2}\right)=x\left(0 ; a_{1}, a_{2}\right) \exp \left(a_{1} t-a_{2} q_{1}(t)\right),
$$




$$
y\left(t ; a_{3}\right)=y\left(0 ; a_{3}\right) \exp \left(\left(q_{2}(t)-a_{3} t\right) .\right.
$$

Now we can express all values of interest with the help of the mgf-s of the initial distributions and the auxiliary variables. The total sizes of prey and predator populations are:

$$
\begin{aligned}
& X(t)=X(0) \int_{A} \exp \left(a_{1} t-a_{2} q_{1}(t)\right) P^{1}\left(0 ; a_{1}, a_{2}\right) d a_{1} d a_{2}=M^{1}\left(t,-q_{1}(t)\right) \\
& Y(t)=Y(0) \int_{A} \exp \left(\left(q_{2}(t)-a_{3} t\right) P^{2}\left(0 ; a_{3}\right) d a_{3}=\exp \left(q_{2}(t)\right) M^{2}(-t)\right.
\end{aligned}
$$

The pdf-s $P^{1}\left(t ; a_{1}, a_{2}\right), P^{2}\left(t ; a_{2}\right)$ are:

$$
\begin{aligned}
& P^{1}\left(t ; a_{1}, a_{2}\right)=x\left(t ; a_{1}, a_{2}\right) / X(t)=P^{1}\left(0 ; a_{1}, a_{2}\right) \exp \left(a_{1} t-a_{2} q_{1}(t)\right) / M^{1}\left(t,-q_{1}(t)\right) \\
& P^{2}\left(t ; a_{3}\right)=y\left(t ; a_{3}\right) / Y(t)=P^{2}\left(0 ; a_{3}\right) \exp \left(-a_{3} t\right) / M^{2}(-t) .
\end{aligned}
$$

The current mean values of the parameters are:

$$
\begin{aligned}
& E_{t}^{1}\left[a_{1}\right]=\frac{\partial}{\partial \lambda_{1}} \ln \left(M^{1}\left(t,-q_{1}(t)\right)\right), \\
& E_{t}^{1}\left[a_{2}\right]=\frac{\partial}{\partial \lambda_{2}} \ln \left(M^{1}\left(t,-q_{1}(t)\right)\right), \\
& E_{t}^{2}\left[a_{3}\right]=\frac{\partial}{\partial \lambda_{3}} \ln \left(M^{2}(-t)\right) .
\end{aligned}
$$

Now we have a closed escort system of non-autonomous equations for the keystone variables:

$$
\begin{aligned}
& d q_{1} / d t=\exp \left(q_{2}(t)\right) M^{2}(-t), \\
& d q_{2} / d t=\frac{\partial}{\partial \lambda_{2}} M^{1}\left(t_{1},-q_{1}\right) .
\end{aligned}
$$

Solving the Cauchy problem (6.12) with zero initial values, we obtain explicit formulas for total populations' sizes (6.9) and current distribution of the parameters (6.10), which completely solve the inhomogeneous version of inhomogeneous model (6.8). One can check that the obtained formulas coincide with the formulas, which follow from Theorem 5 .

Now we are able to compare the initial and inhomogeneous Volterra' prey-predator models. Integrating the equations of system (6.8) over the parameters we obtain the system

$$
\begin{aligned}
& d X / d t=X\left(E_{t}^{1}\left[a_{1}\right]-E_{t}^{1}\left[a_{2}\right] Y\right), \\
& d Y / d t=Y\left(E_{t}^{1}\left[a_{2}\right] X-E_{t}^{2}\left[a_{3}\right]\right) .
\end{aligned}
$$

These equations for total sizes of inhomogeneous populations have the same form as the initial Volterra' system (6.7); the difference is that now the parameter values are not constants but vary over time according to formulas (6.11). The phase-parametric portrait of "homogeneous" Volterra' model is well known (see, e.g., [2]). The dynamics of system (6.12) is determined by the parametric point $\left(E_{t}^{1}\left[a_{1}\right], E_{t}^{1}\left[a_{2}\right], E_{t}^{2}\left[a_{3}\right]\right)$, which moves across the parametric portrait of model (6.7). It means that qualitative behavior of inhomogeneous Volterra model may change with time dependently on the initial distribution, in contrast to the standard version of the model. This phenomenon, which may be referred to as "traveling across the parametric portrait of a homogeneous model" is a common feature of corresponding inhomogeneous models. We have observed it on the example of discrete-time models in s.5 as "traveling along the bifurcation diagram" of corresponding maps. An example of Volterra-type model of two inhomogeneous populations, the "tumor - oncolytic viruses model", is given in Section 8.2.

Other examples of inhomogeneous community models can be found in [28, 43-45]. In the following Sections we will provide specific examples of questions that can now be addressed with the method as it is applied to the models of inhomogeneous communities. 


\section{Preventing the tragedy of the commons through punishment of over- consumers and encouragement of under-consumers}

The conditions that can lead to the exploitative depletion of a shared resource, i.e., the tragedy of the commons [16], can be reformulated as a game of prisoner's dilemma: while preserving the common resource is in the best interest of the group, over-consumption is in the interest of each particular individual at any given point in time. One way to try and prevent the tragedy of the commons is through infliction of punishment for over-consumption and/or encouraging under-consumption, thus selecting against overconsumers. The effectiveness of various punishment functions in an evolving system can be evaluated within a framework of a parametrically heterogeneous consumer-resource model.

Our starting point is a simplified version of the model introduced by Krakauer et al. [36], which is presented in the form

$$
\begin{aligned}
& \frac{d N(t)}{d t}=r N(t)\left(c-\frac{b N(t)}{z(t)}\right), \\
& \frac{d z(t)}{d t}=\gamma-\delta z(t)-\frac{e(1-c) N(t)}{z(t)+N(t)}
\end{aligned}
$$

In this model, consumers $N(t)$ compete with each other for shared resource $z(t)$, which determines the dynamical carrying capacity of the population. The resource is assumed to be renewable in such a way that individuals in the population not only consume the resource but are also able to contribute to its restoration, i.e., they can contribute to increasing the carrying capacity for the entire population.

The per capita birth rate is proportional to the rate $c$ of the resource consumption. The per capita death rate is proportional to $\frac{b N}{z}$, where $b$ characterizes the efficiency of resource consumption. Resource $z(t)$ is restored naturally at constant rate $\gamma \geq 0$, deteriorates at the rate $\delta z, \delta>0$, and can be replenished by the activity of consumers. The rate of restoration of the common resource in response to the activity of individuals is modeled by the function $\frac{e(1-c) N}{z+N}$, where parameter $0<e \leq 1$ denotes the proportion of total resource that is consumed or restored. As the number of consumers increases, the total amount of the shared resource will increase or decrease depending on the value of the parameter $c \geq 0$. More detailed model derivations are given in $[31,33,36]$.

The resource consumption/restoration parameter $c$ is restricted to the interval $c \in \mathbf{A}=[0, a]$. Letting $a \leq 1$ implies that the individuals in the population never consume more than they restore. In contrast with [36], we allow the parameter $c$ to take values in $\mathbf{A}$ as $a>1$; it allows for the presence of overconsumers in the system, so $(1-c)$ can take on negative values, which accounts for strictly consumerist behavior.

Since reduction of the common "carrying capacity" can indeed lead to population collapse, this model allows to model effectively in a conceptual framework the question of the effects of over-consumption on the survival of the population, see [31].

The role of various levels of over-consumption and effectiveness of various punishment functions in an evolving system can be evaluated within a framework of a parametrically heterogeneous consumerresource model [33]. Denote $x_{c}$ to be a set of consumers that are characterized by the same intrinsic and heritable value of parameter $c$. Each individual is punished or rewarded according to a function $f(c)$ that directly affects the fitness of each consumer-producer, such that each clone is immediately punished for over-consumption if $c>1$ or rewarded for under-consumption when $c<1$. Different clones make different contribution to the common resource restoration, so

$$
\frac{d z}{d t}=\gamma-\delta z(t)+e \frac{\int_{\mathbf{A}}(1-c) x_{c}(t) d c}{N(t)+z(t)}=\gamma-\delta z(t)+e \frac{N(t)\left(1-E_{t}[c]\right)}{N(t)+z(t)}
$$

where the total size of the population of consumers is $N(t)=\int_{\mathbf{A}} x_{c}(t) d c$. 
The final form of the parametrically heterogeneous model is now

$$
\begin{aligned}
& \left.\frac{d x_{c}}{d t}=r x_{c}(t)\left(c-\frac{N(t)}{k z(t)}\right)+x_{c}(t) f(c)\right), \\
& \frac{d z}{d t}=\gamma-\delta z(t)+e \frac{N(t)\left(1-E_{t}[c]\right)}{N(t)+z(t)} .
\end{aligned}
$$

Introduce a keystone variable $q(t)$, which satisfies the equation $\frac{d q}{d t}=\frac{N}{k z}$, so that one may rewrite the first equation in the following form:

$$
\frac{d x_{c}}{d t}=r x_{c}(t)\left(c-\frac{d q}{d t}\right)+x_{c}(t) f(c) .
$$

Then $x_{c}(t)=x_{c}(0) e^{-q(t)+t(r c+f(c)}$.

Total population size is then given by

$$
N(t)=N(0) e^{-q(t)} \int_{c} e^{t(r c+f(c))} P(0, c) d c
$$

the current mean value

$E_{t}[c]=\int_{c} c P(t, c) d c$ where

$$
P(t, c)=\int_{c} c e^{t(r c+f(c)) P(0, c) d c} / \int_{c} e^{(t(r c+f(c)) P(0, c) d c} .
$$

As a result, we have now reduced the initial infinitely-dimensional system (7.2) to a two-dimensional system

$$
\begin{aligned}
& \frac{d z}{d t}=\gamma-\delta z+e \frac{N\left(1-E_{t}[c]\right)}{N+z} \\
& \frac{d q}{d t}=\frac{N}{k z}
\end{aligned}
$$

where $N$ and $E_{t}[c]$ are defined by formulas (7.3)-(7.4).

The model was studied analytically in [31] without incorporating punishment/reward for over/under consumption. As the value of $c$ increases, the population goes through a series of transitional regimes from sustainable coexistence with the resource to oscillatory regime to eventually committing evolutionary suicide through decreasing the common carrying capacity to a level that can no longer support the population. Identification of these transitional regimes can then be used as a warning signal, which could foreshadow the upcoming tragedy of the commons and serve as a call for an intervention, and specifically to this example, for an introduction of an appropriate punishment-reward function.

The effectiveness of three types of punishment functions was evaluated in [33]: moderate punishment, $f(c)=a(1-c) /(1+c)$; severe punishment, $f(c)=a(1-c)^{3}$, where the parameter $a$ denotes the severity of implementation of punishment on individuals with the corresponding value of parameter $c$, and function of the type, $f(c)=r\left(1-c^{b}\right)$, which allows to separate the influence of reward for under-consumption, primarily accounted for with parameter $r$, and punishment for overconsumption, accounted for with parameter $b$. It was found that the effectiveness of punishment depends not only on intrinsic parameter values of the system but also on the initial composition of the population. Moreover, when the punishment imposed is moderate, overconsumption could be avoided only when the value of $a$ was very high, i.e. when punishment is imposed very severely. The value of $a$ that was necessary for successful management of over-consumers varied depending on different initial distributions, indicating that in order to be able to prevent the tragedy of the commons, one needs to evaluate not only the type of punishment and the severity of its enforcement but also match it to the composition of the population, since one level of punishment can be effective for one distribution of clones within a population of consumers and not another.

The same set of numerical experiments was conducted for the severe punishment $\backslash$ generous reward function. Then, the value of $a$ that would correspond to successful restraint of over-consumers was much 
lower than in the previous case for all initial distributions considered. The system was able to support individuals with higher values of parameter $c$ present in the initial population than in the previous case. In some cases brief periods of oscillatory transitional dynamical behavior before the system collapsed could be observed.

The punishment function of the type $f(c)=r\left(1-c^{b}\right)$ allows one to account the intensity of punishment and reward by parameters $b$ and $r$ respectively. In order to evaluate the expected effectiveness of the punishment/reward system one needs to not only adjust parameters $b$ and $r$ to each particular case considered but also be able to evaluate the expected range of parameter $c$, since one level of punishment may be appropriate for one set of initial conditions but not another. Moreover, the time to collapse varies depending on the initial distribution of the clones within the population, and the higher the frequency of over-consumers is in the initial population, the worse the prognosis.

Figure 5 shows the dynamics of the total population size and total resource with respect to different values of $a$ (different levels of severity of imposed punishment). One can see that successful management of overconsumers was possible only when punishment implementation was very high.
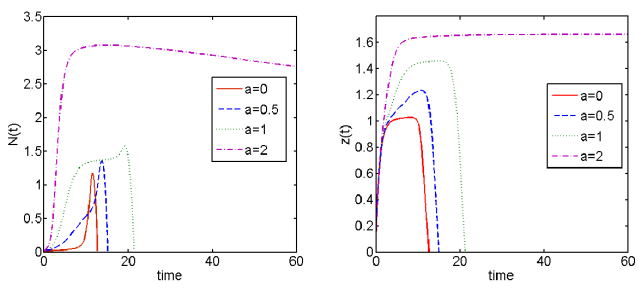

FIGURE 5. The model with severe punishment, $f(c)=a(1-c)^{3}$ and truncated exponential distribution of the parameter $c$. Dynamics of the total population size (left) and total resource (right) with respect to different values of $a$.

Overall, one can conclude not only that the tragedy of the commons can be prevented if the punishment of over-consumers increases non-linearly faster against the rate of over-consumption but also that reward system was inefficient in order to prevent population collapse, and a severe enough of punishment had to be implemented as well.

\section{Tumor modeling}

Another type of a complex evolving system, where heterogeneity cannot be slighted, is cancer, since it is intrinsic genetic intra-tumoral heterogeneity that underlies therapeutic resistance, as cytotoxic therapies wipe out sensitive cell clones, leaving the resistant subpopulation. Heterogeneity thus needs to be taken into account in some way in most of the tumor models in order to render them biologically relevant. There are of course many metrics of heterogeneity that can be chosen when dealing with tumors. Here, we choose to focus on metrics that deal with universal properties of tumor cells, namely, metabolism and resource consumption. We will also consider an example of therapeutic modeling, and specifically, a case of differential sensitivity to oncolytic viruses.

\subsection{Metabolism, glucose and the Warburg effect}

One property of tumor cells that has gained significant attention in the recent years is that of glucose metabolism. Specifically, it has been observed that tumor cells tend to metabolize glucose anaerobically even in the presence of ample oxygen supply. Anaerobic metabolism, or glycolysis, is inefficient in comparison to oxidative phosphorylation, yielding 2 ATP molecules per glucose as opposed to nearly 30 ATPs, which made it ever more intriguing why tumors would persist in this mode of metabolism even when presented with an alternative. 
While this phenomenon was initially observed by Otto Warburg as early as the beginning of the $20^{t h}$ century, which resulted in this property of tumors now being referred to as Warburg effect, the question of why it persists has not been revisited until recently. Otto Warburg's initial hypothesis that mitochondria, which are responsible for the cell's ability to oxidize glucose, are somehow impaired in tumor cells, proved to be incorrect. R. Gatenby and colleagues $[10,11]$ hypothesized that perhaps tumors gain a selective advantage over normal cells by accumulating in the tumor microenvironment lactic acid, which is a byproduct of glycolysis. Therefore, while cancer cells would lose selective advantage by lowering their yield of ATPs, they would gain it through eliminating their competition by secreting toxic waste, i.e., lactic acid.

One issue with this hypothesis lies in the fact that one cancer cell cannot produce enough lactic acid in order to create a sufficiently toxic environment. Many cells need to metabolize glucose anaerobically over an extended period of time in order to the population as a whole to gain a selective advantage. However, before the advantages of glycolysis accumulate, all the cells have to suffer from the consequences of ATP deprivation. One possible way to look at this situation is once again as a prisoner's dilemma: enough cancer cells need to "cooperate" and use glycolysis in order to jointly gain a selective advantage over normal cells. This could become sensible in the case of high initial glucose availability, where inefficiency of glycolysis would not create too high an ATP deprivation. This hypothesis can be evaluated in the following way.

Assume that each cell in the tumor is characterized by a value of parameter $\alpha$, which represents the proportion of total carbon that is used aerobically, thus effectively leaving $(1-\alpha)$ proportion of total carbon for consumption through glycolysis; $x_{\alpha}$ then denotes a set of all cells that are characterized by a fixed heritable value of parameter $\alpha$. The total population size is then taken to be $N(t)=\int_{\mathbb{A}} x_{\alpha} d \alpha$.

Glycolysis is less metabolically efficient and is limited only by glucose supply, denoted by $C^{i n}$; aerobic metabolism is more efficient but is limited both by carbon availability $C^{i n}$ and by oxygen supply, which is accounted for with parameter $\beta$. Each cell $x_{\alpha}$ is thus characterized by its own intrinsic value of $\alpha$, allowing to model population heterogeneity with respect to metabolic strategy.

There are two types of carbon that are taken into account in the model: extracellular carbon and intracellular carbon. Extracellular carbon $C^{e x}$ is replenished in the tissue microenvironment through blood inflow and also is recycled from intracellular stores of cells that have died. It is consumed by the cells, becoming intracellular carbon, based on differences in concentration between $C^{i n}$ and $C^{e x}$. Different cells can consume carbon at different rates: glycolytic cells get less energy per one molecule of glucose, but their rate of carbon uptake is much greater due to upregulation of glucose transporters in the cell membrane. This is accounted for by the parameter

$$
p=p_{a}\left(1-E_{t}[\alpha]\right)+p_{g} E_{t}[\alpha] .
$$

The consumed extracellular carbon is then metabolized by the cells; the higher efficiency of metabolism by aerobic cells is accounted for by the parameter $\xi$.

Taking into account all of these assumptions, we obtain the following System:

$$
\begin{aligned}
& \frac{d x_{\alpha}}{d t}=x_{\alpha}\left(r_{\alpha}(1-\alpha)\left(\frac{\beta C^{i n}}{\beta+C^{i n}}+r_{g} \alpha C^{i n}-d\right),\right. \\
& \frac{d C^{e x}}{d t}=\frac{g_{1}\left(C_{0}-C^{e x}\right)}{N}-\left(p_{g} E_{t}[\alpha]+p_{a}\left(1-E_{t}[\alpha]\right)\right) \frac{\left(C^{e x}-C^{i n}\right)}{k_{1}+C^{e x}-C^{i n}}+C^{i n} d, \\
& \frac{d C^{i n}}{d t}=\left(p_{g} E_{t}[\alpha]+p_{a}\left(1-E_{t}[\alpha]\right)\right) \frac{\left(C^{e x}-C^{i n}\right)}{k_{1}+C^{e x}-C^{i n}}-s C^{i n}\left(r_{\alpha} \xi\left(1-E_{t}[\alpha]\right)+r_{g} E_{t}[\alpha]\right) .
\end{aligned}
$$

Now, let us introduce keystone variables $g(t)$ and $q(t)$, such that

$$
\begin{aligned}
& \frac{d q}{d t}=r_{\alpha} \beta C^{i n} /\left(\beta+C^{i n}\right), \\
& \frac{d g}{d t}=r_{g} C^{i n} .
\end{aligned}
$$

Then $\frac{d x_{\alpha}}{d t}=x_{\alpha}\left((1-\alpha) \frac{d q}{d t}+\alpha \frac{d g}{d t}-\delta\right)$.

Consequently, $x_{\alpha}(t)=x_{\alpha}(0) e^{(1-\alpha) q(t)+\alpha g(t)-\delta t)}$. 
Full population size of cells $x_{\alpha}$ is then given by

$$
N(t)=\int_{A} x_{\alpha}(t) d \alpha=N(0) \int_{A} e^{q(t)-\delta t} e^{\alpha(g(t)-q(t))} P_{0}(\alpha) d \alpha=N(0) e^{q(t)-\delta t} M_{0}(g(t)-q(t)),
$$

where $P_{0}(\alpha)=x_{\alpha}(0) / N(0)$ and where $M_{0}(\lambda)=\int_{0}^{\infty} e^{\lambda \alpha} P_{0}(\alpha) d \alpha$

and where $M_{0}(\lambda)=\int_{0}^{\infty} e^{\lambda \alpha} P_{0}(\alpha) d \alpha$ is the moment generating function (mgf) of the initial distribution of clones $P_{\alpha}(0)$ within the population. The final distribution would then be given by

$$
P_{t}[\alpha]=\frac{x_{\alpha}(t)}{N(t)}=\frac{e^{\alpha(g(t)-q(t)}}{M_{0}[g(t)-q(t)]} .
$$

The full System thus becomes

$$
\begin{aligned}
& \frac{d C^{e x}}{d t}=\frac{g_{1}\left(C_{0}-C^{e x}\right)}{N}-p \frac{\left(C^{e x}-C^{i n}\right)}{k_{1}+C^{e x}-C^{i n}}+C^{i n} d, \\
& \frac{d C^{i n}}{d t}=p \frac{\left(C^{e x}-C^{i n}\right)}{k_{1}+C^{e x}-C^{i n}}-s C^{i n}\left(r_{\alpha} \xi\left(1-E^{t}[\alpha]\right)+r_{g} E_{t}[\alpha]\right), \\
& \frac{d q}{d t}=r_{\alpha} \beta C^{i n} /\left(\beta+C^{i n}\right), \\
& \frac{d g}{d t}=r_{g} C^{i n} .
\end{aligned}
$$

The expected value of $\alpha$ at each time $t$ is calculated through:

$$
E_{t}[\alpha]=\int_{A} \alpha P_{t}(\alpha) d \alpha=\int_{A} P_{0}(\alpha) \frac{\alpha e^{\alpha(g(t)-q(t))}}{M_{0}[g(t)-q(t)]}=\frac{M_{0}^{\prime}[g(t)-q(t)]}{M_{0}[g(t)-q(t)]} .
$$

Through computation of numerical solutions one could observe that increased inflow of extracellular carbon did indeed cause dramatic changes in the composition of cell population over time, more so than changes in oxygen supply (Figure 6). These results suggest that changes in the nutrient availability can indeed support persistence of anaerobic metabolism by decreasing the negative effect of glycolysis, i.e. low ATP yield, but keeping all of its benefits, i.e., better competitive ability. Full investigation of this model is given in [30].

Intriguingly, several prospective studies have shown that mortality from cancer was much lower in those individuals that had higher muscle mass, regardless of their body mass index (BMI), even though the incidence of cancer was the same (see, for instance, [51] and [18]). From the point of view of cell metabolism, this could be due to the fact that muscle cells have higher energy demands than other somatic cells, thus "beating" the glycolytic cells to the nutrients, delaying progression of the disease. So, while exercising will not affect the probability of the person getting cancer in the first place, it may reduce the risk of dying from it.

\subsection{Tumor - oncolytic viruses model}

In this section, we will focus on implications of tumor heterogeneity [1] on therapeutic interventions, highlighting the applicability of HKV-method to this type of problems. Our goal here is to illustrate a very particular application of the method to a population, which is parametrically heterogeneous with respect to two parameters.

In [24] there was formulated and investigated a simplified conceptual model of interactions between tumor cells and an oncolytic virus (oncolytic viruses are viruses that specifically infect and kill cancer cells but do not affect normal cells [40]). Several phenomena that are absent in homogeneous models, such as cancer recurrence, tumor dormancy, and others, appear in heterogeneous setting. Within the applied modeling framework, to overcome the adverse effect of tumor cell heterogeneity on cancer progression, a heterogeneous population of an oncolytic virus must be used. Intrinsic heterogeneity in tumor cell susceptibility to virus infection, as well as the ability of an oncolytic virus to infect tumor cells, can lead to complex quasi-chaotic behavior of the tumor-virus system.

The considered heterogeneous models, although still oversimplified, reflect qualitatively new phenomena, some of which are observed in experiments and clinical trials. The approach was illustrated by 


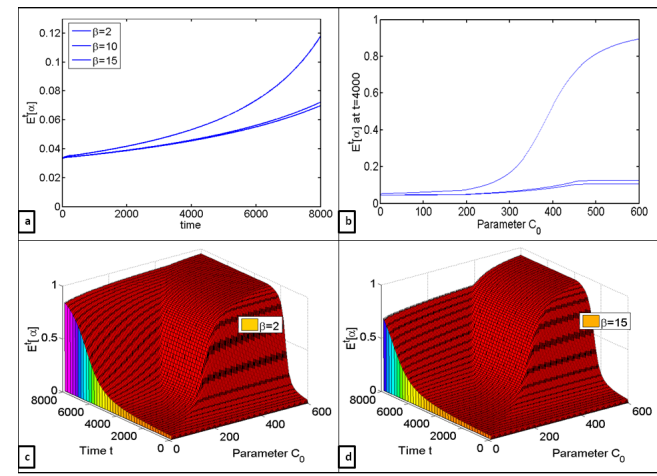

FigURE 6. Quantifying the effects of oxygen availability on the growth of aerobic and glycolytic cell clones. (a) Changes in the mean number of glycolytic cells $E_{t}[\alpha]$ over time for $\beta=2,10,15$ (b) $E_{t}[\alpha]$ at $t=4000$ for $C_{0}$ varied from 5 to 600 , evaluated for $\beta=2,10,15$ (c) Changes in $E_{t}[\alpha]$ over time with respect to differences in $C_{0}$ for $\beta=2$ (d) Changes in $E_{t}[\alpha]$ over time with respect to differences in $C_{0}$ for $\beta=15$.

addressing a complex process that involves both virus-cell interactions and tumor growth, namely, the interaction of the so-called oncolytic viruses with tumors.

Consider the following conceptual model for virus-tumor interactions. Two populations of tumor cells are incorporated here: uninfected tumor cells and infected tumor cells (this is a modification of the model suggested in [35]):

$$
\begin{aligned}
& \frac{d X}{d t}=r_{1} X\left(1-\frac{X+Y}{K}\right)-\frac{b X Y}{X+Y}, \\
& \frac{d Y}{d t}=r_{2} Y\left(1-\frac{X+Y}{K}\right)+\frac{b X Y}{X+Y}-a Y
\end{aligned}
$$

where $X$ is the size of the uninfected cell population; $Y$ is the size of the infected cell population; $r_{1}$ and $r_{2}$ are the maximum per capita growth rates of uninfected and infected cells, respectively; $K$ is the carrying capacity; $b$ is the transmission coefficient (this parameter may also include the replication rate of the virus); and $a$ is the rate of infected cell killing by the virus (cytotoxicity). All the parameters of the model are assumed to be non-negative. The concentration of viral particles is not explicitly included; it is assumed that virus abundance is proportional to infected cell abundance [46].

Re-scaling model (8.5) (see [24] for details) leads to the system

$$
\begin{aligned}
& \frac{d X}{d t}=X(1-(X+Y))-\frac{\beta X Y}{X+Y}, \\
& \frac{d Y}{d t}=\gamma Y(1-(X+Y))+\frac{\beta X Y}{X+Y}-\delta Y
\end{aligned}
$$

where $\beta=b / r_{1}, \gamma=r_{2} / r_{1}, \delta=a / r_{1}$, and $X+Y \leq K=1$. Model (8.6) is subject to initial conditions $X(0)=X_{0}>0$ and $Y(0)=Y_{0}>0$.

The model exhibits all possible outcomes of oncolytic virus infection depending on parameter values, including no effect on the tumor, stabilization or reduction of the tumor load, and complete elimination of the tumor. Moreover, there exist two domains, where the final outcome crucially depends on initial conditions and can result either in failure of virus therapy or in stabilization and elimination of the tumor. The complete phase-parameter portrait of system (8.6) was given in [24].

Now let us assume that the values of parameters of susceptibility $\beta$ and cytotoxicity $\delta$ are not fixed fixed but may take on different values in the cell population. Let $x(t, \beta)$ be the density of uninfected cells having parameter value $\beta$, and $y(t, \delta)$ be the density of infected cells having parameter value $\delta$. Then the dynamics of $y(t, \delta)$ is determined by the equation 


$$
\begin{aligned}
\frac{d y(t, \delta)}{d t} & =y(t, \delta) \gamma\left(1-(X(t)+Y(t))+\frac{y(t, \delta)}{X(t)+Y(t)} \int \beta x(t, \beta) d \beta-y(t, \delta) \delta=\right. \\
& =y(t, \delta)\left[\gamma(1-(X(t)+Y(t)))+\frac{E_{t}[\beta] X(t)}{X(t)+Y(t)}-\delta\right]
\end{aligned}
$$

where $E_{t}[\beta]$ is the mean value of the susceptibility parameter in the uninfected part of the cell population. Then the parametrically heterogeneous version of model (8.2) becomes:

$$
\begin{aligned}
& \frac{d x(t, \beta)}{d t}=x(t, \beta)\left[1-X(t)-Y(t)-\frac{\beta Y(t)}{X(t)+Y(t)}\right], \\
& \frac{d y(t, \delta)}{d t}=y(t, \delta)\left[\gamma(1-X(t)-Y(t))+\frac{E_{t}[\beta] X(t)}{X(t)+Y(t)}-\delta\right],
\end{aligned}
$$

The initial conditions are

$$
x(0, \beta)=x_{0}(\beta)=X_{0} P_{1}(0, \beta), \quad y(0, \delta)=y_{0}(\delta)=Y_{0} P_{2}(0, \delta) .
$$

Integrating both equations in (8.8) over $\beta, \delta$ accordingly, we obtain the following system of ODEs:

$$
\begin{aligned}
& \frac{d X}{d t}=X(1-(X+Y))-E_{t}[\beta] \frac{X Y}{X+Y}, \\
& \frac{d Y}{d t}=\gamma Y(1-(X+Y))+E_{t}[\beta] \frac{X Y}{X+Y}-E_{t}[\delta] Y, \\
& X(0)=X_{0}, \quad Y(0)=Y_{0}
\end{aligned}
$$

where the mean parameter values $E_{t}[\beta], E_{t}[\delta]$ should be determined. Let us define the auxiliary variable

$$
\frac{d q}{d t}=-\frac{Y}{X+Y}, \quad q(0)=0
$$

Then formally

$$
\begin{gathered}
x(t, \beta)=x(0, \beta) \exp \left[\int_{0}^{t}(1-X(u)-Y(u)) d u+\beta q(t)\right], \\
y(t, \delta)=y(0, \delta) \exp \left[\gamma \int_{0}^{t}\left(1-X(u)-Y(u)+\frac{E_{u}[\beta] X(u)}{X(u)+Y(u)}\right) d u-\delta t\right] .
\end{gathered}
$$

Next,

$$
P_{1}(t, \beta)=x(t, \beta) / X(t)=x(t, \beta) / \int x(t, \beta) d \beta=\frac{\exp (\beta q(t))}{M_{\beta}(q(t))} P_{1}(0, \beta)
$$

where $M_{\beta}(q)=\int \exp (q \beta) P_{1}(0, \beta) d \beta$ is the mgf of the initial distribution of the parameter $\beta$. Similarly,

$$
P_{2}(t, \delta)=y(t, \delta) / Y(t)=\frac{\exp (-\delta t)}{M_{\delta}(-t)} P_{2}(0, \delta)
$$

where $M_{\delta}$ is the mgf of the initial distribution of the parameter $\delta$. Hence,

$$
E_{t}[\beta]=\left.\frac{1}{M_{\beta}(q(t))} \frac{d M_{\beta}(\lambda)}{d \lambda}\right|_{\lambda=q_{1}(t)}, \quad E_{t}[\delta]=\left.\frac{1}{M_{\delta}(-t)} \frac{d M_{\delta}(\lambda)}{d \lambda}\right|_{\lambda=-t} .
$$

Now the system (8.9)-(8.11) can be solved for any given initial distributions of the parameters $\beta$ and $\delta$. Unlike the parametrically homogeneous system (8.6), where parameters $\beta$ and $\delta$ remain fixed, in the parametrically heterogeneous system (8.9) $\left\{E_{t}[\beta], E_{t}[\delta]\right\}$ change over time. Therefore, as the expected values change due to system dynamics, the values of $\left\{E_{t}[\beta], E_{t}[\delta]\right\}$ "travel" through the phase-parameter 


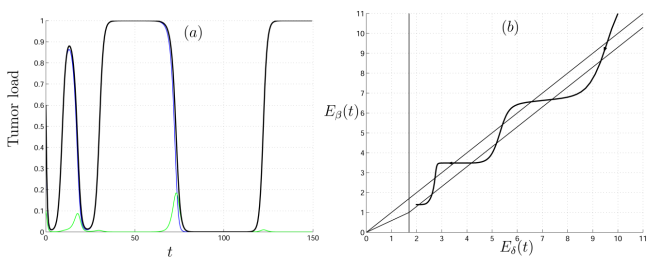

Figure 7. (a) Solutions of system (8.9)-(8.11) with gamma distributed parameters $\beta$ and $\delta$ on $[0, \infty)$. Uninfected cells, $X(t)$, infected cells, $Y(t)$, and the total tumor load, $X(t)+Y(t)$, are shown in blue, green and black, respectively. The initial means of the distributions are $E_{0}[\beta]=11$ and $E_{0}[\delta]=10$, initial variances are $\sigma_{\beta}^{2}=8$ and $\sigma_{\delta}^{2}=0.5$. The initial conditions are $X(0)=0.5, Y(0)=0.1$, and $\gamma=1$. (b) The parametric curve $\left\{E_{t}[\beta], E_{t}[\delta]\right\}$ in the parameter space.

portrait of system (8.6), and can consequently can pass through domains of qualitatively different behavior many times. As a result, much more complex and less predictable and intuitive behavior can be observed, giving insights and predictive power to the model that were not possible with the parametrically homogeneous model.

This can be seen in Figure 6, where the solutions of (8.9)-(8.11) and the path of the mean parameter values are depicted. Moreover, even if the initial and final mean parameter values belong to the same domain, during transient process, the mean parameter values can "visit" other domains (Figure 4).

Some other versions of the considered inhomogeneous model can be found in [24] together with discussion of the results of modeling.

\section{Conclusions}

Replicator equations (RE) are among the basic tools in mathematical theory of selection and evolution. The method of Hidden keystone variables (HKV) for reducing a wide class of RE of high or even infinite dimensionality to low-dimensional escort systems of ODEs, which can be explored analytically in many cases was developed. Notice that even if the analytical solution of the escort system is not available, numerically solving the Cauchy problem for the resulting system of ODEs is much simpler than studying the initial problem numerically. It allows computing (in many cases, explicitly) all statistical characteristics of interest of the initial selection system and visualizing evolutionary trajectories. The approach was developed both to continuous time and discrete time selection systems and replicator equations. The considered examples show how different the global dynamics of a selection system can be depending on the initial distribution even within the frameworks of the same dynamical model.

The HKV method was systematically applied to different inhomogeneous models of populations and communities. Analytical solutions of the considered models can provide new biological insights beyond the computer simulations. The derived explicit solutions may be helpful and necessary in order to be able to completely study corresponding models, which belong to different areas of mathematical biology. Applications to some ecological problems [21,23, 25], global demography [22], cancer modeling [24,30], epidemics in heterogeneous populations [42,43,45], ecological niche construction [31], modeling of the tragedy of commons [33], etc. were published recently. It is our hope that the developed approach has potential for different applications in these and others areas of science.

Acknowledgements. G.K. was supported by the Intramural Research Program of the NIH, NCBI. I.K. was partially supported by the Office of Science (BER), U.S. Department of Energy, under Award Number DE-SC0001434 (to Philip Hahnfeldt, her postdoctoral fellowship mentor). 


\section{References}

[1] R. Alexandrova. Tumour heterogeneity. Exper. Path. \& Paras., 4 (2001), 57-67.

[2] A. D. Bazykin. Nonlinear dynamics of interacting populations. World Scientific, 1998.

[3] F. Berezovskaya, G. Karev, T. W. Snell. Modeling the dynamics of natural rotifer populations: phase-parametric analysis. Ecol. Complex., 2 (2005), 395-409.

[4] F. Brauer, C. Castillo-Chavez. Mathematical models in population biology and epidemiology. Springer, 2011.

[5] A. Cintron-Arias, F. Sanchez, X. Wang, C. Castillo-Chavez, D. M. Gorman, P. J. Gruenewald. The role of nonlinear relapse on contagion amongst drinking communities. In: Chowell, G., Hayman, J.M., Bettencourt, L.M.A., CastilloChavez, C. (Eds.), Mathematical and statistical estimation approaches in epidemiology, Springer Netherlands, (2009), 343-360.

[6] T. Dietz, E. Ostrom, P. C. Stern. The struggle to govern the commons. Science, 302 (2003), $1907-1912$.

[7] W. Feller. An introduction to probability theory and its applications. Vol. 2. John Wiley \& Sons, 2008.

[8] R. Fisher. The genetical theory of natural selection: a complete variorum edition. Oxford University Press, 1999.

[9] H. Von Foerster, P. M. Mora, L. W. Amiot. Doomsday: Friday, 13 November, ad 2026. Science, 132 (1960), $1291-1295$.

[10] R. A. Gatenby, T. L. Vincent. Application of quantitative models from population biology and evolutionary game theory to tumor therapeutic strategies. Mol. Can. Therap., 2 (2003), 919-927.

[11] R. A. Gatenby, R. J. Gillies. Why do cancers have high aerobic glycolysis? Nature Rev. Can., 4 (2004), 891-899.

[12] G. Gause. The struggle for existence. Courier Dover Publications, 2003.

[13] A. N. Gorban. Equilibrium encircling. Equations of chemical kinetics and their thermodynamic analysis, Nauka, Novosibirsk, 1984.

[14] A. N. Gorban. Selection theorem for systems with inheritance. Math. Mod. Nat. Phen., 2 (2007), 1-45.

[15] J. Hofbauer, K. Sigmund. Evolutionary games and population dynamics. Cambridge University Press, 1998.

[16] Hardin, Garrett. The tragedy of the commons. Science, 162 (1968), 1243-1248.

[17] G. H. Heppner, F. R. Miller. The cellular basis of tumor progression. Int. Rev. Cytol., 177 (1997), 1-56.

[18] F.B. Hu, W. C. Willett, T. Li, M. J. Stampfer, G. A. Colditz, J. E. Manson. Adiposity as compared with physical activity in predicting mortality among women. N. Eng. J. Med., 351 (2004), 2694-2703.

[19] S. Kapitza. The phenomenological theory of world population growth. Physics-Uspekhi, 39 (1996), 57-71.

[20] S. Kapitza. Global Population Blow-Up and After: The Demographic Revolution and Information Society. Global Marshall Plan Initiative, 2006.

[21] G. P. Karev. Inhomogeneous models of tree stand self-thinning. Ecol. Mod., 160 (2003), 23-37.

[22] G. P. Karev. Dynamics of inhomogeneous populations and global demography models. J. Biol. Sys., 13 (2005), 83-104.

[23] G. P. Karev, A.R. Burk. Analytical models of forest dynamics in stable environment. In: New developments in ecology research. Nova Science Publishers, New York, USA (2006), 29-97.

[24] G. P. Karev, A. S. Novozhilov, E. V. Koonin. Mathematical modeling of tumor therapy with oncolytic viruses: effects of parametric heterogeneity on cell dynamics. Biol. Dir., 1 (2006), 19.

[25] G. P. Karev, A. S. Novozhilov, F. S. Berezovskaya. Modeling the dynamics of inhomogeneous natural rotifer populations under toxicant exposure. Ecol. Mod., 212, (2008), 80-85.

[26] G. P. Karev. Inhomogeneous maps and mathematical theory of selection. Journal of Difference Equations and Applications, 14.1 (2008), 31-58.

[27] G. P. Karev. On mathematical theory of selection, continuous time population dynamics. J. Math. Bio., 60 (2010), 107-129.

[28] G. P. Karev. Principle of minimum discrimination information and replica dynamics. Entropy, 12 (2010), $1673-1695$.

[29] G. P. Karev, A. S. Novozhilov, F. S. Berezovskaya. On the asymptotic behaviour of the solutions to the replicator equation. Math. Med. \& Biol., 28 (2011), 89-110.

[30] I. Kareva. Prisoner's Dilemma in Cancer Metabolism. PloS one, 6.12 (2011), e28576.

[31] I. Kareva, F. Berezovskaya, C. Castillo-Chavez. Transitional regimes as early warning signals in resource dependent competition models. Math. Biosci., 240 (2012), 114-123.

[32] I. Kareva, F. Berezovskaya, C. Castillo-Chavez. Myeloid cells in tumour - immune interactions. J. Biol. Dyn., 4 (2010), 315-327.

[33] I. Kareva, B. Morin, G. Karev. Preventing the tragedy of the commons through punishment of over-consumers and encouragement of under-consumers. Bull. Math. Biol., 75, (2013), 565-588.

[34] G. F. Khilmi. Foundations of the Physics of the Biosphere, 1967.

[35] N. Komarova. Mathematical modeling of tumorigenesis: mission possible. Curr. Opin. Oncol., 17 (2005),39-43.

[36] D.C. Krakauer, K. M. Page, D. H. Erwin. Diversity, dilemmas, and monopolies of niche construction. Am. Nat., 173 (2009), 26-40.

[37] Y. Kuang, J. D. Nagy, J. J. Elser. Biological stoichiometry of tumor dynamics: mathematical models and analysis. Disc. Cont. Dyn. Sys. Ser. B, 4 (2004), 221-240.

[38] Y.A. Kuznetsov. Elements of applied bifurcation theory, Springer-Verlag, New York, 1995.

[39] J. Liebig. Chemistry applications to farming and physiology, 1876.

[40] F. McCormick. Cancer specific viruses and the development of ONYX-015. Canc. Biol. \& Ther., 2 (2003), $156-159$. 
[41] A. S. Novozhilov, F. S. Berezovskaya, E. V. Koonin, G. P. Karev. Mathematical modeling of tumor therapy with oncolytic viruses: regimes with complete tumor elimination within the framework of deterministic models. Biol. Dir., 1 (2006), 18.

[42] A. S. Novozhilov. On the spread of epidemics in a closed heterogeneous population. Math. Biosci., 215 (2008), $177-185$.

[43] A. S. Novozhilov. Epidemiological models with parametric heterogeneity: Deterministic theory for closed populations. Math. Mod. Nat. Phen., 7 (2012), 147-167.

[44] A. S. Novozhilov. Analysis of a generalized population predator-prey model with a parameter distributed normally over the individuals in the predator population. J. Comp. Sys. Sci. Int., 43 (2004), 378-382.

[45] A. S. Novozhilov. Heterogeneous Susceptibles-Infectives model: Mechanistic derivation of the power law transmission function. Dyn. Cont., Disc. \& Impul. Sys., Ser. A, Math. Anal., 16 (2009), 136-140.

[46] M. Nowak, R. M. May. Virus Dynamics: Mathematical Principles of Immunology and Virology: Mathematical Principles of Immunology and Virology. Oxford University Press, 2000.

[47] E. Ostrom. Coping with tragedies of the commons. Ann. Rev. Pol. Sci., 2 (1999), 493-535.

[48] I.A. Poletaev. On mathematical models of elementary processes in biogeocoenosis. Problems of Cibernetics, 16 (1966), 171-190.

[49] T.W. Snell, M. Serra. Dynamics of natural rotifer populations. Hydrobio., 368 (1998), 29-35.

[50] A. Tsoularis, J. Wallace. Analysis of logistic growth models. Math. Biosci., 179 (2002), 21-55.

[51] M. E. Wright, S. C. Chang, A. Schatzkin, D. Albanes, V. Kipnis, T. Mouw, P. Hurwitz, A. Hollenbeck, M. F. Leitzmann. Prospective study of adiposity and weight change in relation to prostate cancer incidence and mortality. Cancer, 109 (2007), 675-684.

[52] V.V. Zagreev. Reference Book on All-Union Forest Growth Tables. Kolos, Moscow, 1992.

[53] K. B. Zeldovich, P. Chen, B. E. Shakhnovich, E. I. Shakhnovich. A first-principles model of early evolution: emergence of gene families, species, and preferred protein folds. PLoS Comp. Biol., 3 (2007), e139. 